\title{
Photoprotective Effects of Soybean Extract against UV-Induced Damage in Human Fibroblast and Hairless Mouse Model
}

\author{
Young-Chang Cho,", Jae-Bok Han ${ }^{2, \#}$ and Sang-Ik Park ${ }^{3, *}$ \\ ${ }^{1}$ College of Pharmacy, Chonnam National University, Gwangju 61186, Korea \\ ${ }^{2}$ Department of Radiological Science, Dongshin University, Naju 58245, Korea \\ ${ }^{3}$ College of Veterinary Medicine, Chonnam National University, Gwangju 61186, Korea
}

Received January 17, 2019

Revised January 30, 2019

Accepted Febuary 8, 2019

\section{*Correspondence}

Sang-lk Park

College of Veterinary Medicine, Chonnam

National University, 77 Yongbong-ro, Buk-

gu, Gwangju 61186, Korea

Tel: $+82-62-530-2843$

Fax: +82-62-530-0835

E-mail: sipark@jnu.ac.kr

ORCID

https://orcid.org/0000-0003-1709-0324

"Young-Chang Cho and Jae-Bok Han contributed equally to this work.

\begin{abstract}
Soy isoflavones have been reported to possess many physiological activities such as antioxidant activity and inhibition of cancer cell proliferation. This study investigated the photoprotective effects of soybean extract in human fibroblast cell line and hairless mice model. Human fibroblast was treated with soybean extract before and after ultraviolet B (UVB; 290-302 nm) irradiation. In the soybean extract treated group, the cells showed better resistance to ultraviolet (UV) than control group. The amount of type I collagen recovered from the soybean treated group was higher than the vehicle group exposed to UV-induced damage. Moreover, increased expression of metalloproteinases-1 as a result of UV irradiation was suppressed by the soybean extract. Female mice were orally administered soybean extract and irradiated with UVB light for 8 weeks. The effects of the soybean extract on the skin appearance, collagen deposition and epidermal thickness in the UV-damaged mouse skin were analyzed using histopathological methods. In soybean extract treated group, the skin had a better morphology than that of the control group. Furthermore, the amount of type I collagen was increased and overexpression of MMP-1 was reduced in the soybean extract group compared to vehicle group. Additionally, up-regulation of pro-inflammatory cytokines induced by UV irradiation was suppressed by dietary soybean extract treatment. It appears that soybean extract had a photoprotective effect, including anti-aging and anti-inflammatory effect, from UV-induced damage in not only human fibroblast, but also hairless mice. We confirmed that these effects were possibly due to promotion of collagen synthesis and inhibition of MMP-1 expression.
\end{abstract}

Keywords: anti-aging effect, isoflavone, photoprotective effect, soybean extract

\section{INTRODUCTION}

Ultraviolet (UV) radiation, especially UVB (280-320 nm) from sunlight, is one of the major environmental perils to induce skin injury. Edema, erythema, hyperpigmentation, hyperplasia, photoaging, inflammation, DNA damage and mutations in the skin were caused by UV exposure (de Gruijl and Forbes, 1995; Afaq and Mukhtar, 2006; Huang et al., 2010). Moreover, it has been reported that longterm exposure to UV radiation raised the risk of skin cancer (Godar, 2005; Huang et al., 2010).

In human skin, levels of various matrix metallopro- 
teinases (MMPs) including MMP-1, MMP-3, and MMP-9 were raised by UV irradiation (Fisher et al., 1996; Brenneisen et al., 2002; Shin et al., 2017). Particularly, MMP1 , as a potent fibroblast collagenase, is highly responsible for degrading dermal components in the extracellular matrix (ECM) (Iyer et al., 2006; Dong et al., 2008; Shin et al., 2017). When increased MMP-1 level induces breaking down of fibrillary type I and III collagen, advanced processing is followed by MMP-3 and MMP-9 (Hessler et al., 1997; Shin et al., 2017). Hence, MMP-1 plays an essential role in the initiation of ECM degradation caused by UV irradiation. Upregulation of the mitogen-activated protein kinase (MAPK) signaling pathway promotes MMP1 overexpression via various elements such as cytokines and growth factor receptors (Brenneisen et al., 2002; Shin et al., 2017). When MAPK pathway regulates expression of MMPs, activator protein (AP)-1 acts as a transcription factor and a major effector. Then, AP-1 proceeds to formation of heterodimer complexes together with Jun, Fos or activating transcription factor proteins (Karin et al., 1997; Shin et al., 2017). Consequently, acute or chronic exposure to UV can stimulate photoaging, the inhibition of MMP-1 expression can be a critical factor in photoprotective effect (Shin et al., 2017).

Soy foods for nutritional or medical purpose have been steadily developed and consumed in Asian countries (Messina et al., 2006). Soybean has been acknowledged as a high-quality protein source, since it contains several number of physiologically active components (Lokuruka, 2011). Among these compounds, isoflavones are related to helpful effects on human health, with anti-carcinogenic properties and estrogen-like effects based on their diphenolic structure (Setchell, 1998; Sarkar and Li, 2003).

12 different isoforms of soy isoflavones have been found and can be classified into four chemical forms based on physiological activity; aglycone (daidzein, genistein and glycitein), glucoside (daidzin, genistin and glycitin), acetylglucoside (acetyldaidzin, acetylgenistin and acetylglycitin), and malonylglucoside (malonyldaidzin, malonylgenistin and malonylglycitin). Many bioactive function of soy isoflavones have been found like important role in antioxidant (Kao and Chen, 2006: Huang et al., 2010), suppression of cancer cell propagation (Kao et al., 2007a), anti-inflammatory activities (Kao et al., 2007b; Huang et al., 2010), inhibition of coronary heart diseases (Dalais et al., 2003; Huang et al., 2010), as well as osteoporosis (Mi- gliaccio and Anderson, 2003; Huang et al., 2010). In antiinflammatory function, soy isoflavones could diminish the secretions of interleukin-1 (IL-1), IL-6, nitric oxide (NO) and prostaglandin E2 (PGE2) in the cell supernatant and fluid of mouse peritoneal exudate (Kao et al., 2007b; Huang et al., 2010).

Therefore, the aims of this study were to prepare soybean extract, which contains optimal amount of soy isoflavones, and to evaluate the anti-aging and photoprotective effects on human fibroblast and in hairless mice.

\section{MATERIALS AND METHODS}

\section{Chemicals and reagents}

Dulbecco's modified eagle medium (DMEM) was purchased from Welgene (Gyeongsan, Korea). FBS was purchased from Gibco (Waltham, MA, USA). 3-[4,5-dimethylatiazol-2-yl]-2,5 diphenyltetrazolium bromide (MTT) powder and dimethyl sulfoxide were purchased from Sigma-aldrich (St. Louis,, USA). Penicillin-Streptomycin solution was purchased from Mediatech, Inc. (Manassas, VA, USA). Human COL1A1 enzyme-linked immune sorbent assay (ELISA) kit was purchased from CUSABIO (Wuhan, China).

\section{Preparation of soybean extracts}

Soybeans (100 g) were extracted by ethanol in 18 conditions at various alcohol contents, temperature and time with or without sonication; for alcohol $60 \%, 70 \%$ and $100 \%$ were used, for temperature $4^{\circ} \mathrm{C}, 40^{\circ} \mathrm{C}, 80^{\circ} \mathrm{C}$ and $100^{\circ} \mathrm{C}$ were used, for time $3 \mathrm{hr}, 5 \mathrm{hr}, 3$ days and 5 days were used. Under designated condition combined ethanol extracts were dried in a vacuum desiccator under reduced pressure and concentrated using a rotavapor. Concentrated soybean extracts were used after resolving in dimethyl sulfoxide at desired concentration and DMSO were used as a vehicle.

\section{Cell culture}

Human fibroblasts Hs68 were attained from the American Type Culture Collection (ATCC, Manassas, VA) and maintained in Dulbecco's modified Eagle's medium (DMEM, Welgene, Korea) supplemented with FBS (10\%, v/v) and antimycotic-antibiotics (1\%). The cells were incubated at $37^{\circ} \mathrm{C}$ in a $5 \% \mathrm{CO}_{2}$, and $90 \%$ air humidified incubator. The cells were grown on $60 \mathrm{~mm}$ culture dishes to until 
$80 \%-90 \%$ of confluenecy.

\section{Animals and treatments}

Six-week-old female hairless mice, SKH-1 mice, were provided by Da-Mul science (Dae-Jeon, Korea). Mice were acclimated for 2 week prior to the study and supplied with water and food freely. Three to four mice were allocated into 4 groups. The test compounds (soybean extract, $200 \mathrm{mg} / \mathrm{kg} / \mathrm{day}$ ) and vehicle (1x PBS) and positive isoflavone (genistein, $30 \mathrm{mg} / \mathrm{kg} /$ day) were orally administered for 8 weeks. Body weight and food intake were monitored on a weekly basis. This experimental design was approved by the Institutional Animal Care and Use Committee of Chonnam National University.

\section{Cell viability}

Cell cytotoxicity was performed by MTT assay. Human fibroblast cells were cultured in 96-well plates at a density of $2 \times 10^{3}$ cells/well and incubated in DMEM-10\% FBS containing penicillin/streptomycin at $37^{\circ} \mathrm{C}$ in a $5 \% \mathrm{CO}_{2}$ atmosphere. Cells were treated with each desired chemicals after starvation in serum-free DMEM for $24 \mathrm{~h}$. The cells and each sample were incubated for $24 \mathrm{~h}$ at $37^{\circ} \mathrm{C}$, followed by treatment with MTT solution for $2 \mathrm{~h}$. The cell were washed with PBS and formazan crystals were dissolved by the addition of $100 \mu \mathrm{L}$ of dimethyl sulfoxide (DMSO) in each well. The absorbance at $570 \mathrm{~nm}$ was then measured using a microplate reader (Molecular Devices, Sunnyvale, CA, USA) and the soybean extract-treated and non-treated cells were compared.

\section{UVB irradiation}

Human fibroblast and SKH-1 mice were subject to UVBinduced photoagingas described previously. UVB irradiation device that included a TL20W/12RS UV lamp (Philips, Eindhoven, The Netherlands) with an emission spectrum between 280 and $320 \mathrm{~nm}$ (average $302 \mathrm{~nm}$ ) served as the UV source. Fibroblast cells were irradiated $60 \mathrm{~mJ} / \mathrm{cm}^{2}$ of UVB dose for $10 \mathrm{~min}$ at one time. SKH-1 mice were exposed to UVB by $2 \mathrm{MEdD}\left(2 \mathrm{MEdD}=120 \mathrm{~mJ} / \mathrm{cm}^{2}\right), 5$ times a week for 8 weeks.

\section{Histological evaluation}

To evaluate epidermal thickness, hematoxylin and eosin staining was done Mouse skin samples were fixed with $10 \%$ neutral-buffered formalin, and embedded in paraffin.
Serial sections $(3 \mu \mathrm{m})$ were performed on slides. After deparaffinizing, skin sections were re-hydrated and stained with hematoxylin solution for $3 \mathrm{~min}$, followed by washing for 10 min and dehydration for counterstaining with eosin Y solution for $1 \mathrm{~min}$. Next, the slides were dehydrated through 95\% alcohol and washed in absolute alcohol for 5 min each. Lastly, the slides were cleared in xylene for 20 min to remaining water. Skin sections were examined at $200 \times$ magnification using Olympus AX70 light microscope (Tokyo, Japan).

\section{Real-Time quantitative PCR}

Primary human fibroblast cells were treated with soybean extract for $24 \mathrm{~h}$ and harvested in RNAiso Plus (Takara Bio Inc., Shiga, Japan). RNA was quantified using a NanoDrop ND-2000 spectrophotometer (Thermo Fisher Scientific, Waltham, MA, USA). After reverse transcription with oligo-dT primers using a PrimeScript ${ }^{\mathrm{TM}} 1$ st strand cDNA synthesis kit (Takara Bio Inc.), real-time quantitative polymerase chain reaction (PCR)( was performed using IQ SYBR (Bio-Rad Laboratories) and $2 \mu \mathrm{L}$ of cDNA in triplicate with glyceraldehyde 3-phosphate dehydrogenase (GAPDH) as an internal control. Amplification consisted of 44 cycles at $95^{\circ} \mathrm{C}$ for $10 \mathrm{~s}, 60^{\circ} \mathrm{C}$ for $30 \mathrm{~s}$, and $72^{\circ} \mathrm{C}$ for 30 s. PCR was performed with a CFX Connect ${ }^{\mathrm{TM}}$ Real-Time PCR Detection System (Bio-Rad Laboratories, Hercules, CA, USA). cDNA was probed with the following primers: (Table 1).

\section{Enzyme-linked immunosorbent assay (ELISA) for measuring type 1 collagen synthesis}

Human fibroblast cells were seeded in 96-well plates at a density of $5 \times 10^{3}$ cells/well in a medium containing $10 \%$ FBS for $24 \mathrm{~h}$. After confirming cell attachment, vehicle, genistein and soybean extract was added in FBS-

Table 1. Primer pairs used for real-time polymerase chain reaction in this study

\begin{tabular}{lccc}
\hline \multicolumn{2}{c}{ Gene } & \multicolumn{1}{c}{ Primer sequence } & Product size \\
\hline GAPDH & Forward & 5'-AACGGATTTGGTCGTATTGG-3' & 116 \\
& Reverse & 5'-TGGAAGATGGTGATGGGATT-3 & \\
TNF- $\alpha$ & Forward & 5'-ATTTATTAACGTACTGTCA-3' & 196 \\
& Reverse & 5'-AGACGCCTGCTGATTTAT-3' & \\
IL-1 $\beta$ & Forward & 5'-ATTTGGGGATTGGGCATCC-3' & 243 \\
& Reverse & 5'-GCGTGAGATGGCACGTAA-3' & \\
\hline
\end{tabular}


free medium at concentrations of $0.1 \%, 3 \mu \mathrm{M}$ and $30 \mu \mathrm{M}$, respectively, before and after UVB irradiation. Type-1 collagen production was measured as the release of extracellular matrix protein into the culture medium. A 100$\mu \mathrm{L}$ aliquot of cultured medium and standards (supplied by the kit) was inserted into collagen-coated 96-well plates and incubated at $37^{\circ} \mathrm{C}$ for $2 \mathrm{~h}$. After washing with $0.05 \%$ phosphate-buffered saline with $0.1 \%$ Tween 20 (PBST), COL1A1 primary antibody (supplied by the kit) was added and this was incubated for $1 \mathrm{~h}$. After washing with $0.05 \%$ PBST, the secondary antibody conjugated with HRP (supplied by the kit) was added and incubated for 2 hours at RT. After washing with $0.05 \%$ PBST, the tetramethylbenzidine (TMB) substrate was added. TMB substrate yields a blue color when it catalyzes with the HRP enzyme. After the desired color intensity was acquired, we added 0.5 $\mathrm{N} \mathrm{H} 2 \mathrm{SO} 4$ and finished the reaction and observed color change from blue to yellow. The absorbance was read at $450 \mathrm{~nm}$ with the ELISA machine.

\section{Enzyme-linked immunosorbent assay (ELISA) for measuring MMP1}

Hs68 cells were cultured in a 96-well plate $\left(4 \times 10^{4}\right.$ cells/well) and pretreated with soybean extract, vehicle or genistein for $24 \mathrm{hr}$. The cells were washed with PBS, irradiated with UVB $\left(60 \mathrm{~mJ} / \mathrm{cm}^{2}\right)$ through a thin layer of PBS after washing, and then incubated with serum-free DMEM. After 24 h, MMP-1 in culture medium was collected and quantified by using a human MMP-1 ELISA kit (QIA55; Merck \& Co. Inc., Whitehouse Station, NJ, USA) according to the manufacturer's instructions. First, protein in the supernatant were coated in the microplate with coating buffer for overnight at $4^{\circ} \mathrm{C}$. Each sample was washed with PBST 3 times, for blocking 1\% BSA solution was added and incubated for $2 \mathrm{hr}$ at $37^{\circ} \mathrm{C}$. After washing with PBST 3 times, 1 -microliters of HRP-Avidin was added and incubated for $1 \mathrm{hr}$ at $37^{\circ} \mathrm{C}$. TMB subsrate was added and incubated for $30 \mathrm{~min}$ at room temperature. When $\mathrm{NaOH}$ was added to the sample, it was measured by ELISA reader at $450 \mathrm{~nm}$ wavelength.

\section{Statistical analysis}

Data are presented as mean values and standard errors of the mean (S.E.M) of between three and four independent experiments performed in triplicate. The data were confirmed for normality. Statistical analysis was carried out using One-way analysis of variance (ANOVA) and Tukey's multiple comparison test. Differences were considered significant if $p<0.05$.

Table 2. Various alcohol extracting conditions (18 independent conditions) for determination of the optimal soy isoflavone extract from $100 \mathrm{~g}$ of soybean (Sun-Yu bean from Jeollanam-do province)

\begin{tabular}{|c|c|c|c|c|c|}
\hline \multirow{2}{*}{$\#$} & \multirow{2}{*}{ Condition } & \multirow{2}{*}{ Temperature } & \multirow{2}{*}{ Time duration } & \multicolumn{2}{|c|}{ Isoflavones $(\mathrm{mg} / 100 \mathrm{~g}$ of soybean) } \\
\hline & & & & Glucoside & Aglycones \\
\hline 1 & $60 \% \mathrm{EtOH}$ & $80^{\circ} \mathrm{C}$ & $3 \mathrm{hr}$ & 1004.7 & 27.4 \\
\hline 2 & $60 \% \mathrm{EtOH}$ & $80^{\circ} \mathrm{C}$ & $5 \mathrm{hr}$ & 1112.2 & 30.1 \\
\hline 3 & $60 \% \mathrm{EtOH}$ & $4^{\circ} \mathrm{C}$ & 3 days & 1204.1 & 33.0 \\
\hline 4 & $60 \% \mathrm{EtOH}$ & $4^{\circ} \mathrm{C}$ & 5 days & 1247.2 & 37.0 \\
\hline 5 & $60 \%$ EtOH sonication & $40^{\circ} \mathrm{C}$ & $3 \mathrm{hr}$ & 268.3 & 90.3 \\
\hline 6 & $60 \%$ EtOH sonication & $40^{\circ} \mathrm{C}$ & $5 \mathrm{hr}$ & 298.5 & 77.7 \\
\hline 7 & $70 \% \mathrm{EtOH}$ & $80^{\circ} \mathrm{C}$ & $3 \mathrm{hr}$ & 600.1 & 67.0 \\
\hline 8 & $70 \% \mathrm{EtOH}$ & $80^{\circ} \mathrm{C}$ & $5 \mathrm{hr}$ & 874.5 & 94.3 \\
\hline 9 & $70 \% \mathrm{EtOH}$ & $4^{\circ} \mathrm{C}$ & 3 days & 842.4 & 49.7 \\
\hline 10 & $70 \% \mathrm{EtOH}$ & $4^{\circ} \mathrm{C}$ & 5 days & 1033.7 & 27.1 \\
\hline 11 & $70 \%$ EtOH sonication & $40^{\circ} \mathrm{C}$ & $3 \mathrm{hr}$ & 777.7 & 41.1 \\
\hline 12 & $70 \%$ EtOH sonication & $40^{\circ} \mathrm{C}$ & $5 \mathrm{hr}$ & 951.6 & 51.1 \\
\hline 13 & $100 \% \mathrm{EtOH}$ & $100^{\circ} \mathrm{C}$ & $3 \mathrm{hr}$ & 756.3 & 29.0 \\
\hline 14 & $100 \% \mathrm{EtOH}$ & $100^{\circ} \mathrm{C}$ & $5 \mathrm{hr}$ & 448.0 & 57.0 \\
\hline 15 & $100 \% \mathrm{EtOH}$ & $4^{\circ} \mathrm{C}$ & 3 days & 106.5 & 33.0 \\
\hline 16 & $100 \% \mathrm{EtOH}$ & $4^{\circ} \mathrm{C}$ & 5 days & 171.3 & 41.4 \\
\hline 17 & $100 \%$ EtOH sonication & $40^{\circ} \mathrm{C}$ & $3 \mathrm{hr}$ & 1440.5 & 17.7 \\
\hline 18 & $100 \%$ EtOH sonication & $40^{\circ} \mathrm{C}$ & $5 \mathrm{hr}$ & 1005.9 & 43.1 \\
\hline
\end{tabular}




\section{RESULTS}

\section{Determination of optimal condition for extracting soybean isoflavone}

In the alcohol extract with $100 \mathrm{~g}$ of soybean, we compared 18 extraction conditions. Between conditions, there were some statistical differences for total isoflavones and amount of aglycones at different hr of extraction time without agitation (Table 2).

According to the results, isoflavone extraction from soybean flours could be efficiently achieved by using ethanol $70 \%$ as solvent for 5 hours at $80^{\circ} \mathrm{C}$. The average total isoflavone concentration was $9.63 \mathrm{mg} / 100 \mathrm{~g}$, and total glucoside, including didzin, glycitiin and genistin, was 874.52 $\mathrm{mg} / 100 \mathrm{~g}$ and total aglycone, including daidzein, glycitein and genistein was $8.87 \mathrm{mg} / 100 \mathrm{~g}$. We used this optimal condition for further experiment.

\section{Cell viability test for determination of efficacy does of soybean extract}

Cell viability was $95-100 \%$ when treated with the soybean extract as described above, which contains both the optimal amount of total aglycone and acetylglucoside forms of isoflavones. In order to explore the cytotoxicity effects of soybean extract on fibroblast cells, cells were treated with the soybean extract (Vehicle, 6, 12, 25, 100 and $200 \mu \mathrm{g} / \mathrm{mL}$ ). The result showed that soybean extract is non-toxic for human fibroblast until $100 \mu \mathrm{g} / \mathrm{mL}$ (Fig. 1).

\section{Effect of soybean extract on type 1 collagen synthesis in cultured fibroblast cells}

Destruction of collagen and elastic fibers is mainly responsible for skin-aging and enlarged skin pores (Katsuta et al., 2005; Roh et al., 2006; Son et al., 2013). Type 1 collagen synthesis was measured by ELISA to evaluate the skin elasticity effect of soybean extract. When 10 and 100

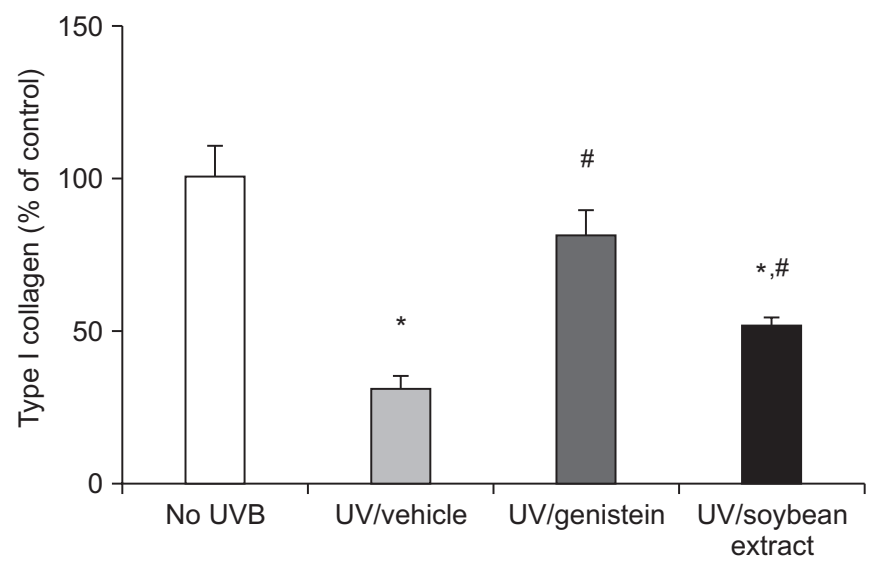

Fig. 2. The effect of soybean extract on type-l collagen synthesis in human fibroblasts. Type 1 collagen synthesis was measured by ELISA to evaluate the skin elasticity effect of soybean extract at concentration of $100 \mu \mathrm{g} / \mathrm{mL}$ and compared with positive control group treated with genistein at concentration of $3 \mu \mathrm{M}$, before and after UVB irradiation. No UVB, no UVBirradiation (as a negative control), UV/Vehicle, UVB-irradiated and treated with DMSO. Data was represented as the mean \pm S.E.M.; $n=3 ;{ }^{*}, p<0.05$ compared to the UV, \#, $p<0.05 \mathrm{com}^{-}$ pared to UV/Vehicle.
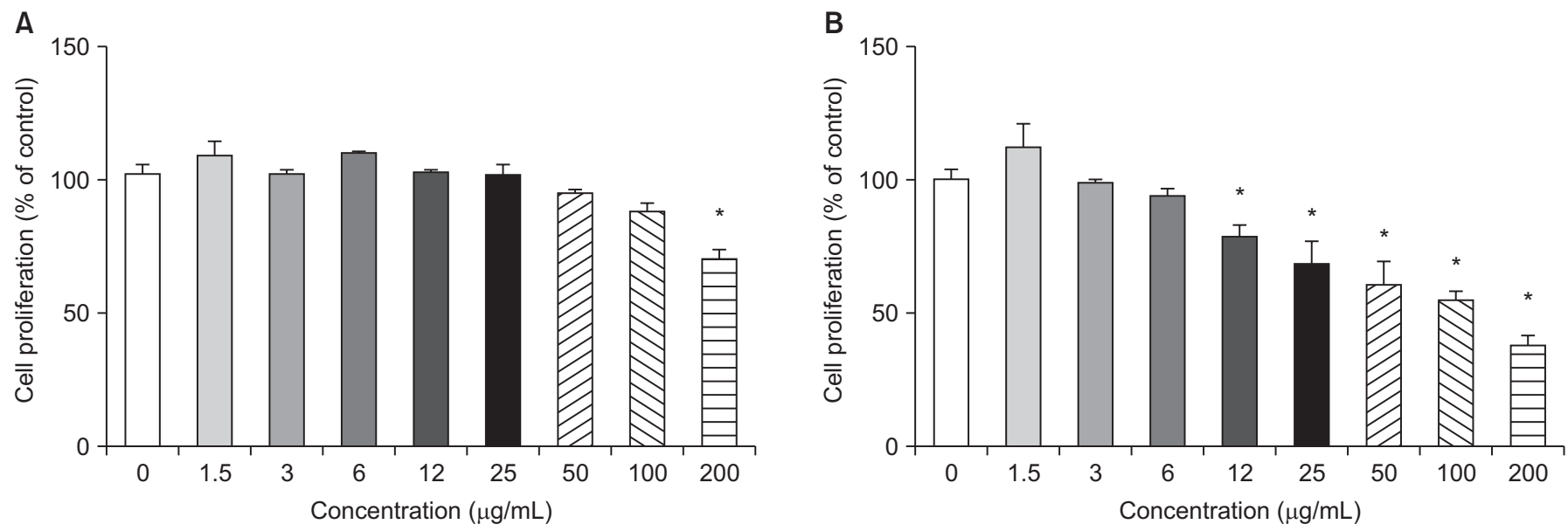

Fig. 1. Effects of each soybean extract (A) and genistein (B) on the suppression of the proliferation of human fibroblast HS68 cells via 3-(4,5-dimethyl- thiazol-2-yl)-2,5-diphenyltetra zolium bromide (MTT) assay. Cell were exposed to the indicated concentration of test compounds for $48 \mathrm{hr}$. Control cells were exposed only solvent (dimethyl sulfoxide, DMSO). Results are expressed as a percentage of the solvent control, which were expressed as a percentage of the solvent control (set as 100\%), and each value was represented as the mean \pm S.E.M. of three independent experiments; ${ }^{*} p<0.05$ compared to the corresponding control according to ANOVA test followed by Turkey's multiple-comparison test. 
$\mu \mathrm{g} / \mathrm{mL}$ soybean extract were imposed on fibroblast cells for $24 \mathrm{~h}$, the concentrations were not toxic according to MTT assay. Hence soybean extract promoted type 1 collagen synthesis in a dose-dependent manner. The $100 \mu \mathrm{g} /$ $\mathrm{mL}$ of soybean extract treatment yielded $50 \%$ increased type 1 collagen synthesis, and the positive vitamin $\mathrm{C}$ control gave a $150 \%$ increase in type 1 collagen synthesis (Fig.

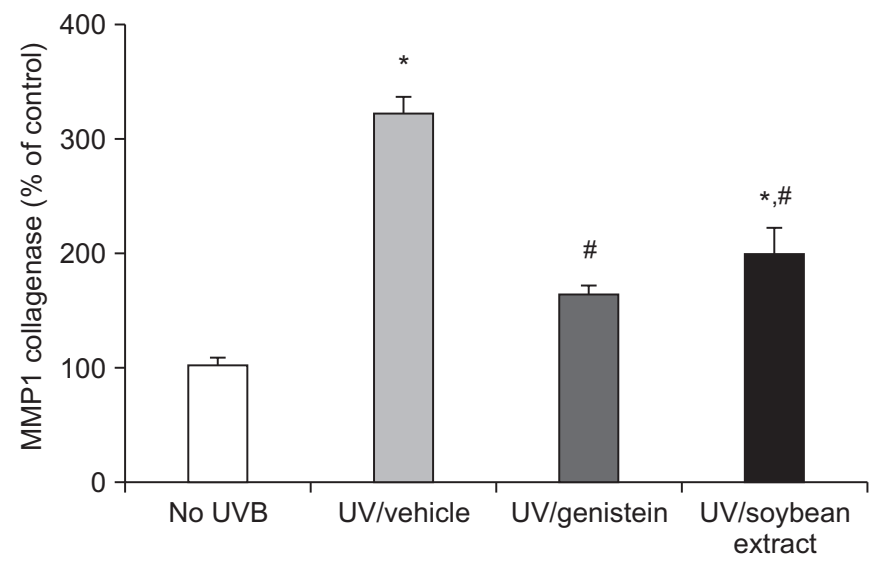

Fig. 3. Effect of soybean extract on MMP-1 expression via ELISA in cultured fibroblast medium. Cells were treated with vehicle (DMSO), genistein $(6 \mu \mathrm{M})$ or soybean extract $(100 \mu \mathrm{g} / \mathrm{mL})$ before UVB irradiation and maintained with treatment after irradiation. The supernatant was collected 48 hours later. The contents of MMP-1 in supernatant were determined according to the instructions of ELISA kit, and the results were read using the microplate reader, which was used to reflect the expression of MMP-1 in senescent cells. Data are expressed as the mean \pm S.E.M of three independent experiments $(\mathrm{n}=3) .{ }^{*}, p<0.05$ compared to the UV, \#, $p<0.05$ compared to UV/Vehicle.
2) after UVB-irradiation. The soybean extract- treated group showed significant high level of type 1 collagen compared to no UVB-irradiation group. These results suggest that soybean extract recover injury in fibroblast from UV irradiation.

\section{Soybean extract suppresses UVB-induced MMP-1 overexpression in cultured fibroblasts}

To elucidate the molecular mechanisms underlying the anti-wrinkling effects of soybean extract, an in vitro study was performed. Soybean extract at the concentration of $100 \mu \mathrm{g} / \mathrm{mL}$ was observed to significantly reduce MMP-1 protein expression after UVB-irradiation (Fig. 3). These inhibitory effects of soybean extract were apparent within a concentration range that did not affect cell viability.

\section{Clinical observation and histopathological observations}

During the study period, the body weight of the hairless mice was measured regularly. Mean body weights of the nonirradiated control group, UV-irradiated control group and soybean extract group were similarly increased throughout the period of study. There was no significant difference in the mean body weight among the groups (data not shown). Food consumption during the experimental period was not different among the three groups (data not shown). Therefore, it seems that body weight gain and food consumption were not affected by UV irradiation and soybean extract treatment.

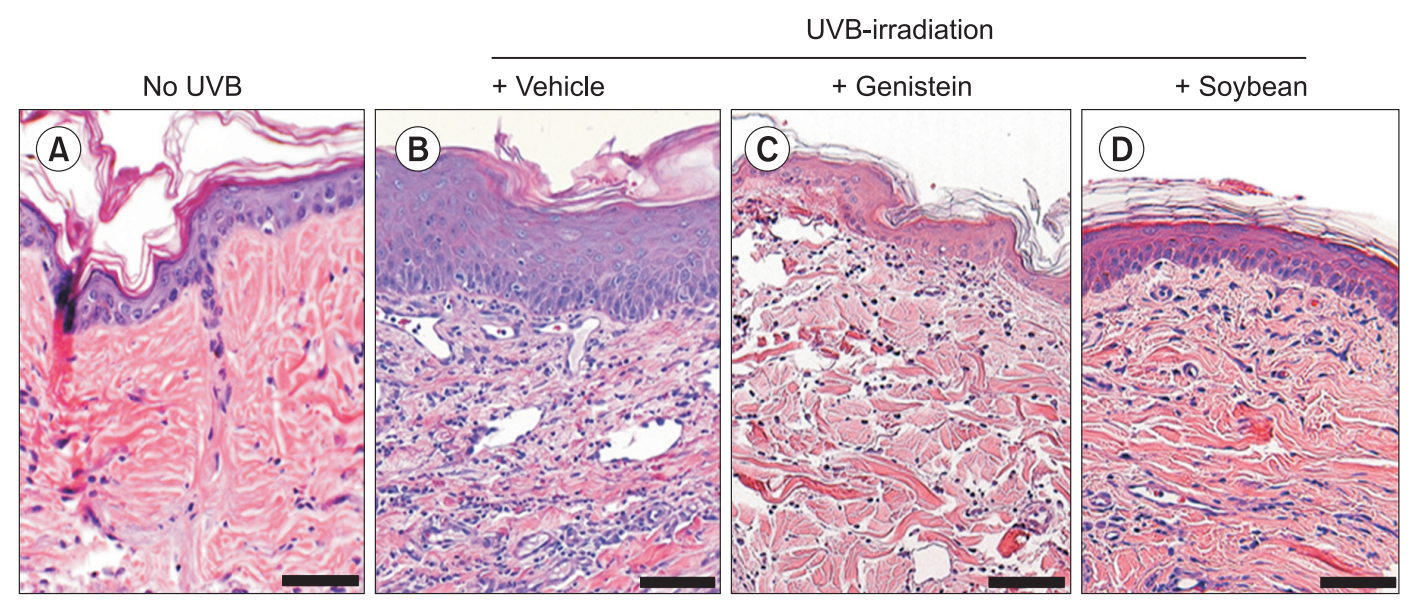

Fig. 4. Histological observation on SKH-1 hairless mice skin treated with vehicle, genistein or soybean extract for 8 weeks. Hematoxylin and eosin stain (H\&E). Scale bar $=100 \mu \mathrm{m}$. UVB-irradiation was performed daily, 5 times a week, at $2 \mathrm{MEdD}(2 \mathrm{MEdD}=120 \mathrm{~mJ} /$ $\mathrm{cm}^{2}$ ) during experiment. (A) age-matched, normal mouse skin. (B) Skin of mice UV-irradiated for 8 weeks. (C) Skin of mice orally administrated with genistein at $20 \mathrm{mg} / \mathrm{kg} /$ day, 2 times a day. (D) Skin of mice orally administrated with soybean extract at $200 \mathrm{mg} / \mathrm{kg} /$ day, 2 times a day. The influence of the isoflavones on the dermis, epidermis, collagen and fibroblasts was investigated. 


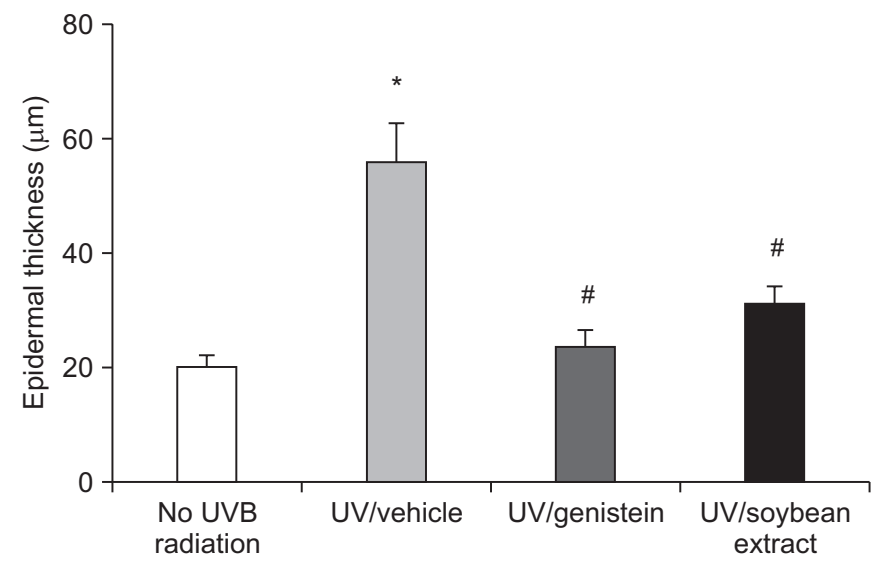

Fig. 5. Photoprotective effect of soybean extract against UV in hairless mice. The mice were UV-irradiated 5 times per week for 8 weeks as described in Materials and Methods. The epidermal thickness was measured by ImageJ program on the $H$ \& E stained sections. The results are expressed as a mean S.E.M. of the thickness $(\mu \mathrm{m})$. Values were measured from 3-independet experiment. NO UVB radiation, age-matched normal control without irradiation for 8 weeks, UV/Vehicle, group orally administrated with PBS after daily irradiation. UV/Genistein, group orally administrated with genistein $(20 \mathrm{mg} / \mathrm{kg} /$ day $)$ after daily irradiation. UV/Soybean extract, group orally administrated with soybean extract $\left(200 \mathrm{mg} / \mathrm{kg} /\right.$ day) after daily irradiation. ${ }^{*}, p<$ 0.05 compared to no UVB, \#, $p<0.05$ compared to UV/Vehicle.

For histopathological analysis, UV irradiated hairless mice showed greater changes in the epidermis than in the age-matched normal mice (Fig. 4, 5). Fig. 5 shows the histological measurements of the epidermal thickness of the hairless mouse skin. Measurements of the epidermal thickness showed significant increases in both the UV control group and the isoflavone group compared to the age-matched normal group. UV irradiation induced a $170 \%$ increase in the epidermal thickness relative to the age matched normal group after four weeks irradiation. However, the epidermal thickness of the soybean extract treated group was $87 \%$ of that of the UV irradiated control group. The mean epidermal thickness in the soybean extract group $(23.73 \pm 2.25 \mu \mathrm{m})$ was significantly thinner than in the UV control group $(52.31 \pm 6.04 \mu \mathrm{m})$.

\section{Soybean prevents UVB-induced collagen degradation in hairless mice}

When human skin is acutely exposed to UVB, epidermal hyperplasia (Kajiya et al., 2009) and collagen degradation occurs (Choi et al., 2007). To determine the effect of UVB exposure on epidermal thickness and collagen degradation, mouse skin samples were collected and subject to

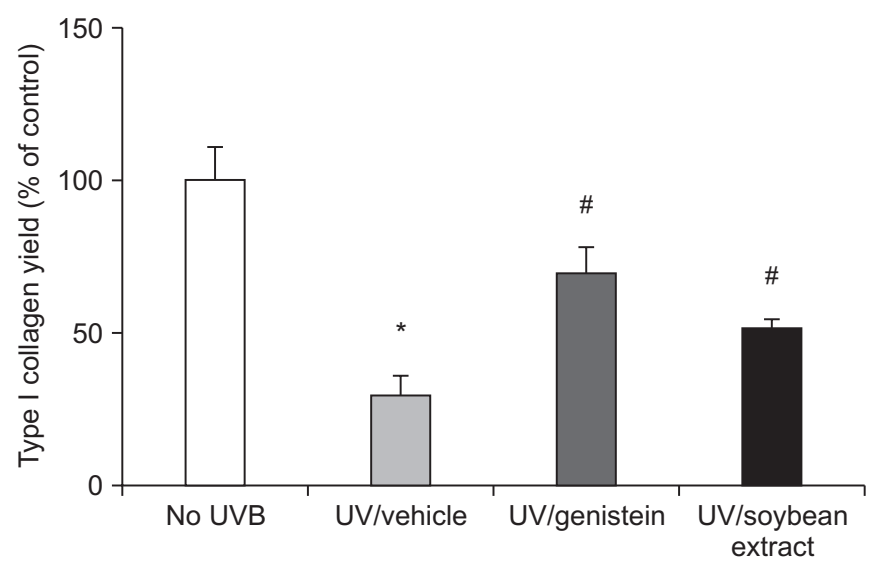

Fig. 6. The effect of soybean extract on type-I collagen synthesis in SKH hairless mice with UVB irradiation. The group treated with genistein and soybean extract showed significant increase of type I collagen after UV radiation, indicating photoprotective effect from UV. ${ }^{*}, p<0.05$ compared to no UVB, $\#, p<0.05$ compared to UV/Vehicle.

homogenization vigorously with beads in PBS. The supernatant was analyzed after centrifugation.

As a greater decrease in epidermal thickness was observed for the soybean extract group compared to the animals that received only vehicle (PBS), type 1 collagen levels were also recovered in the treatment groups (Fig. 6).

\section{Soy isoflavone extract suppresses the expression of COX-2 after UVB exposure}

Cyclooxygenase-2 (COX-2) is inducible and can be induced by multiple mitogenic and inflammatory stimuli, such as hormones, growth factors, cytokines, tumor promoters and UV light. The physiological functions mediated by COX-2 include fever, inflammation, pain, vasodilation, angiogenesis and increased vascular permeability (Sarkar and Li, 2003; Lokuruka, 2011).

Next to elucidate the molecular mechanisms underlying anti-inflammatory effects of soybean extract, we checked if soybean extract suppresses UVB-induced proinflammatory cytokines up-regulation in hairless mice. As soybean extract was observed to prevent degradation of type I collagen expression after UVB-irradiation, this group effectively suppressed UVB-induced up-regulation of TNF- $\alpha$ and IL-1 $\beta$ mRNA level (Fig. 7). These inhibitory effects of soybean were apparent within a concentration range that did not affect cell viability (Fig. 1). 
A

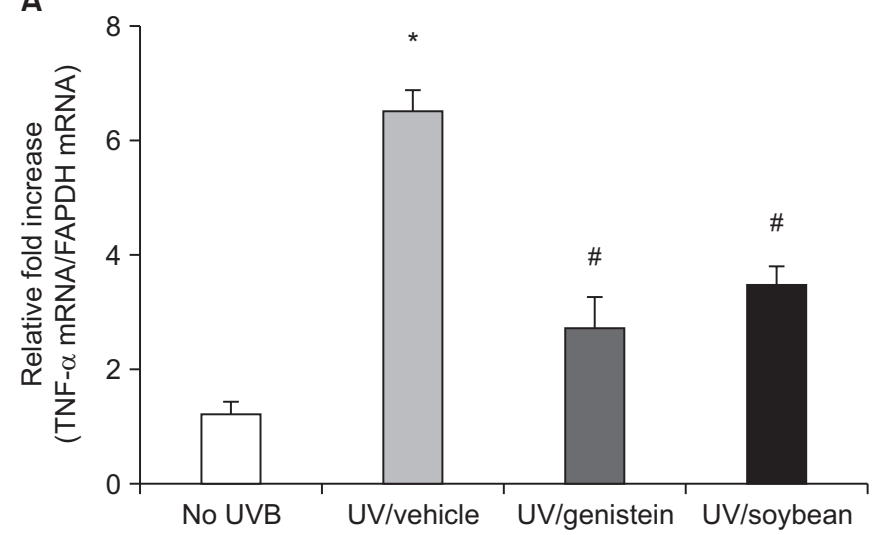

B

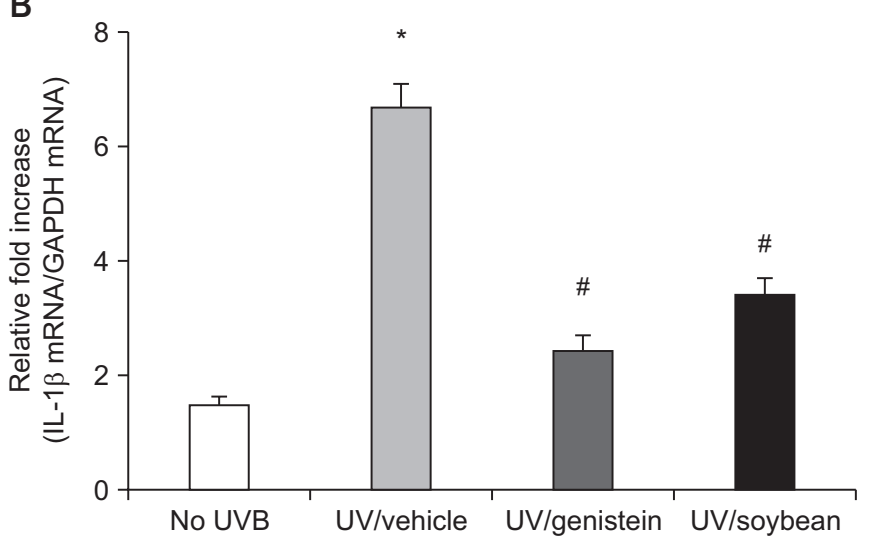

Fig. 7. The effect of soybean extract on regulation of 2 major pro-inflammatory cytokines in SKH mice via quantitative real-time PCR, mRNA levels of TNF- $\alpha(A)$ and IL-1 $\beta$ (B). The group treated with genistein and soybean extract showed significant down-regulation of pro-inflammatory cytokines induced by UV irradiation. Effect of anti-inflammation was effectively observed. ${ }^{*}, p<0.05$ compared to no UVB, \#, $p<0.05$ compared to UV/Vehicle.

\section{DISCUSSION}

UV-induced damage on skin is on account of reactive oxygen species (ROS), which break down anti-oxidative defense mechanism of our body in enzymatic or nonenzymatic pathway. ROS result in oxidative damage to cellular and non-cellular constituents, inhibition of skin regeneration and immunosuppression in skin (Miyachi, 1995). Particularly, $\mathrm{H}_{2} \mathrm{O}_{2}$, leadingly induced by UV light, is a major causative ROS of oxidative stress and skin cell damage (Chiang et al., 2007). Moreover, all these consequence could increase the risk of skin cancer as a chronic pathological change after long-term exposure to UV according to an epidemiologic study (Godar, 2005). Once UV exposure facilitates expression of cyclooxygenase-2 (COX-2), following increased activities of mitogen-activated protein kinase (MAPK) and p38 could contribute to cutaneous inflammation and even carcinogenesis (Podda et al., 1998). In hairless mice model, UV exposure is responsible for acute sunburn and skin aging in acute and chronic pathological changes, respectively (Wei et al., 2003).

Considering that UV stimulates oxidative damages to the skin, the regular intake of antioxidants and antioxidant nutrients or reducing photoaging effect are recommended to be a useful way to reduce the adverse effects of UV radiation (Chiang et al., 2007). In that purpose, soy isoflavones are considered to be an important target, because they have positive effects on the skin via multiple mechanisms such as the prevention of lipid oxidation, stimulation of fibroblast proliferation, reduction of collagen degradation.

In this study, the photoprotective effects of soybean extract on the skin were investigated. As described above, soy isoflavones are composed of 12 different isoforms and can be categorized into four chemical forms: aglycone (daidzein, genistein and glycitein), glucoside (daidzin, genistin and glycitin), acetylglucoside (acetyldaidzin, acetylgenistin and acetylglycitin), and malonylglucoside (malonyldaidzin, malonylgenistin and malonylglycitin). Among these isoforms, aglycones are considered to physiologically have a potent function. Based on alcohol extraction, we compared different extracting condition (different time point and temperature, Table 2) and determined the optimal condition for extraction. From $100 \mathrm{~g}$ of soybean, we acquired $96.3 \mathrm{mg} / 100 \mathrm{~g}$ of total aglycones (didzein, glycitein, genistein) and $874.52 \mathrm{mg} / 100 \mathrm{~g}$ of total glucosides (didzin, glycitiin and genistin). We maintained this extracting condition, $70 \% \mathrm{EtOH}, 5 \mathrm{hr}$, for further bioactive study.

In vitro study, we applied effective dose of soybean extract in human fibroblast cell, and we found the soybean extract treated group showed significantly high level of type 1 collagen compared to no UVB-irradiation group (Fig. 2). These result suggests that soybean extract can heal injury in fibroblast from UV irradiation

To check collagenase activity, we performed ELISA for measuring MMP1 level from supernatant and we confirmed soybean extract suppressed UVB-induced MMP-1 overexpression in cultured fibroblasts (Fig. 3). 
To check the effect of soybean extract in vivo study, we investigated the positive effects of orally administrated soybean extract on UV-induced skin aging using a hairless mouse model for the first. These results demonstrated that the dietary soybean extract have photoprotective effects in a hairless mouse model.

As shown in previous results, UV irradiation induced an increase in the number of inflammatory cells and facilitated epidermal hyperplasia in the hairless mice (Fig. 4, 5). However, the soybean extract treated group had a better skin cell morphology than that of control group according to the histopathological findings.

One major reason for skin damage or destruction is denaturation of collagen and elastic fibers (Katsuta et al., 2005; Roh et al., 2006). Therefore, Type 1 collagen synthesis was also checked by ELISA to gauge the skin elasticity effect of soybean extract. Intake of soybean extract at the concentration of $100 \mathrm{mg} / \mathrm{kg} /$ day stimulated type 1 collagen synthesis, showing $50 \%$ more increase of type 1 collagen synthesis compared to vehicle group after UVBirradiation. The positive genistein control gave a $150 \%$ increase in type 1 collagen synthesis after UVB-irradiation (Fig. 6).

Up-regulation of the transcription of MMP encoding genes are reportedly caused by UV-induced ROS (Miyachi, 1995). The MMPs are a family of proteolytic enzymes that particularly destroy collagen, elastin and other proteins in the connective tissue and bone. Once UV light activated transcription factor activation protein 1 (AP-1), and nuclear factor-kappa B (NF-KB), the transcription of the genes for the MMPs are up-regulated (Varani et al., 2001). A free radical scavenger most probably prevent UVinduced dermal injury by ameliorating the initiation of the MMPs. Retinoic acid, which is reportedly capable of restoring photo-aged skin, has been found to inhibit the UV/ROS induced signal transduction pathway for MMPs activation (Fisher et al., 1996). It has recently shown that pretreatment of genistein prevented c-Jun protein and its driven enzymes, such as collagenase, after UV exposure, which is derived from suppressing the tyrosine kinase and anti-oxidant activities (Kang et al., 2003). Therefore, soybean extract which is capable of antioxidant activities could partially preclude from photo-aging by functioning as free radical scavengers. So we performed checking pro-inflammatory cytokine level by qPCR, as an effect of the soybean extract on the AP-1 and NF-kB signal path- ways. We found that soybean extract effectively suppress of induction of pro-inflammatory cytokines (Fig. 7).

Soy isoflavones have been revealed to retain many bioactive functions such as antioxidant (Kao and Chen, 2006; Huang et al., 2010), prevention of cancer cell proliferation (Kao et al., 2007a), suppression of proinflammatory activities (Kao et al., 2007b; Huang et al., 2010), amelioration of coronary heart diseases (Dalais et al., 2003; Huang et al., 2010), as well as osteoporosis (Migliaccio and Anderson, 2003; Huang et al., 2010). In respect to suppressing pro-inflammatory effects, soy isoflavones could diminish the secretions of various of mediators such as interleukin-1 (IL-1), IL-6, nitric oxide (NO) and prostaglandin E2 (PGE2) in the cell supernatant and fluid of mouse peritoneal exudate (Kao et al., 2007b; Huang et al., 2010). As our hypothesis, we confirmed that soy isoflavones from soybean extract can have positive effects on the skin and collagen layer, then they could greatly contribute to e management of skin health which is mainly initiated by estrogen insufficiency.

\section{CONFLICTS OF INTEREST}

No potential conflict of interest relevant to this article was reported.

\section{ACKNOWLEDGEMENTS}

This research was financially supported by the Ministry of SMEs and Startups (MSS), Korea, under the "Regional Specialized Industry Development Program (R\&D, R0006331)" supervised by the Korea Institute for Advancement of Technology (KIAT).

\section{REFERENCES}

Afaq F and Mukhtar H. 2006. Botanical antioxidants in the prevention of photocarcinogenesis and photoaging. Exp. Dermatol. 15:678-684.

Brenneisen P, Sies H and Scharffetter-Kochanek K. 2002. Ultraviolet-B irradiation and matrix metalloproteinases: From induction via signaling to initial events. Ann. N. Y. Acad. Sci. 973:31-43.

Chiang HS, Wu WB, Fang JY, Chen BH, Kao TH, Chen YT, Huang CC and Hung CF. 2007. UVB-protective effects of isoflavone extracts from soybean cake in human keratinocytes. Int. J. Mol. Sci. 8:651-661.

Choi MS, Yoo MS, Son DJ, Jung HY, Lee SH, Jung JK, Lee BC, 
Yun YP, Pyo HB and Hong JT. 2007. Increase of collagen synthesis by obovatol through stimulation of the TGF-signaling and inhibition of matrix metalloproteinase in UVB-irradiated human fibroblast. J. Dermatol. Sci. 46:127-137.

Dalais FS, Ebeling PR, Kotsopoulos D, McGrath BP and Teede HJ. 2003. The effects of soy protein containing isoflavones on lipids and indices of bone resorption in postmenopausal women. Clin. Endocrinol. (Oxf.) 58:704-709.

de Gruijl FR and Forbes PD. 1995. UV-induced skin cancer in a hairless mouse model. Bioessays 17: 651-660.

Dong KK, Damaghi N, Picart SD, Markova NG, Obayashi K, Okano Y, Masaki H, Grether-Beck S, Krutmann J and Smiles KA. 2008. UV-induced DNA damage initiates release of MMP-1 in human skin. Exp. Dermatol. 17:1037-1044.

Fisher GJ, Datta SC, Talwar HS, Wang ZQ, Varani J, Kang S and Voorhees JJ. 1996. Molecular basis of sun-induced premature skin ageing and retinoid antagonism. Nature. 379: 335-339.

Godar DE. 2005. UV doses worldwide. Photochem. Photobiol. 81:736-749.

Hessler PE, Larsen PE, Constantinou AI, Schram KH and Weber JM. 1997. Isolation of isoflavones from soy-based fermentations of the erythromycin-producing bacterium Saccharopolyspora erythraea. Appl. Microbiol. Biotechnol. 47: 398-404.

Huang CC, Hsu BY, Wu NL, Tsui WH, Lin TJ, Su CC and Hung CF. 2010. Anti-photoaging effects of soy isoflavone extract (aglycone and acetylglucoside form) from soybean cake. Int. J. Mol. Sci. 12:4782-4795.

Iyer S, Visse R, Nagase H and Acharya KR. 2006. Crystal structure of an active form of human MMP-1. J. Mol. Biol.362: 78-88.

Kajiya K, Sawane M, Huggenberger R and Detmar M. 2009. Activation of the VEGFR-3 pathway by VEGF-C attenuates UVB-induced edema formation and skin inflammation by promoting lymphangiogenesis. J. Investig. Dermatol. 129: 1292-1298.

Kang S, Chung JH, Lee JH, Fisher GJ, Wan YS, Duell EA and Voorhees JJ. 2003. Topical N-acetyl cysteine and genistein prevent ultraviolet- light-induced signaling that leads to photoaging in human skin in vivo. J. Invest. Dermatol. 120:835-841.

Kao TH, Huang RF and Chen BH. 2007. Antiproliferation of hepatoma cell and progression of cell cycle as affected by isoflavone extracts from soybean cake. Int. J. Mol. Sci. 8: 1095-1110.

Kao TH, Wu WM, Hung CF, Wu W.B and Chen BH. 2007. Antiinflammatory effects of isoflavone powder produced from soybean cake. J. Agric. Food Chem. 55: 11068-11079.
Kao TH and Chen BH. 2006. Functional components in soybean cake and their effects on antioxidant activity. J. Agric. Food Chem. 54:7544-7555.

Karin M, Liu Z and Zandi E. 1997. AP-1 function and regulation. Curr. Opin. Cell Biol. 9: 240-246.

Messina M, Nagata C and Wu AH. 2006. Estimated Asian adult soy protein and isoflavone intakes. Nutr. Cancer 55:1-12.

Katsuta Y, Iida T, Inomata S and Denda M. 2005. Unsaturated fatty acids induce calcium influx into keratinocytes and cause abnormal differentiation of epidermis. J. Invest. Dermatol. 124,1008-1013.

Lokuruka M. 2011. Effects of processing on soybean nutrients and potential impact on consumer health: An overview. Afr. J. Food Agric. Nutr. Dev. 11.

Migliaccio S and Anderson JJ. 2003. Isoflavones and skeletal health: Are these molecules ready for clinical application? Osteoporos Int. 14:361-368.

Miyachi Y. 1995. Photoaging from an oxidative standpoint. J. Dermatol. Sci. 9:79-86.

Podda M, Traber MG, Weber C, Yan LJ and Packer L. 1998. UVirradiation depletes antioxidants and causes oxidative damage in a model of human skin. Free Radic. Biol. Med. 24: 55-65.

Roh M, Han M, Kim D and Chung K. 2006. Sebum output as a factor contributing to the size of facial pores. Br. J. Dermatol., 155:890-894.

Sarkar FH and Li Y. 2003. Soy isoflavones and cancer prevention: Clinical science review. Cancer Investig. 21:744-757.

Setchell KD. 1998. Phytoestrogens: The biochemistry, physiology, and implications for human health of soy isoflavones. Am. J. Clin. Nutr. 68: 1333S-1346S.

Shin JE, Kim JE, Pak KJ, Kang JI, Kim TS, Lee SY, Yeo IH, Park JHY, Kim JH, Kang NJ and Lee KW. 2017. A combination of soybean and haematococcus extract alleviates ultraviolet Binduced photoaging. Int. J. Mol. Sci. 18: 682-694.

Son DH, Nam MH, Hong CO, Seol HM, Yang JE, Kim YB, Kim CT and Lee KW. 2013. 5- $\alpha$ reductase inhibitory effect and astringent activity of green apple rind extract on human keratinocytes and fibroblast cells. Biosci. Biotechnol. Biochem. 77: 714-721.

Varani V, Spearman D, Perone P, Fligiel SE, Datta SC, Wang ZQ, Shao Y, Kang S, Fisher GJ and Voorhees JJ. 2001. Inhibition of type I procollagen synthesis by damaged collagen in photoaged skin and by collagenase-degraded collagen in vitro. Am. J. Pathol.158:931-942.

Wei H, Saladi R, Lu Y, Wang Y, Palep SR, Moore J, Phelps R, Shyong E and Lebwohl MG. 2003. Isoflavone genistein: Photoprotection and clinical implications in dermatology. J. Nutr. 133: 3811S-3819S. 